\title{
Research on Risk Management Organization of Subway Construction Project Based on Social Network Analysis
}

\author{
Chao Yuan \\ Tianjin University of Technology, Tianjin 300384, China
}

13821655242@163.com

Keywords: Social Network Analysis, Subway Construction Project, Risk Management Organization.

\begin{abstract}
This article introduces the social network analysis method to analyze the risk management organization of subway construction project. From the two aspects of the whole network and the individual network, this paper analyzes the network density and centrality of the social network structure of subway construction project to clarify the overall structure of the risk management organization and the role of each participating party. The study found that the overall structure of the risk management organization in the subway construction project is not close enough. The rights and resources in the network are concentrated in a few participants such as the owner, the contractor and the insurance broker, while the remaining organizations have limited influence on risk management. According to the result of social network analysis, this paper puts forward the optimization suggestion of risk management organization.
\end{abstract}

\section{Introduction}

In recent years, the increasing problem of traffic congestion in urban areas has become a huge obstacle to the sustainable development of economy and society. As an underground transportation system, the subway can effectively relieve the pressure of urban traffic and enhance the image of the city[1].In 2006, China clearly proposed the new requirement of "accelerating the construction of urban rail transit" in the "11th Five-Year Plan", marking that cities have entered a new era of subway[2]. Subway construction has entered a stage of rapid development, but the high risk in the process of subway construction can not be ignored. The particularity of the subway construction project makes its management and risk control extremely complicated. The probability of a safety accident during construction is very high[3]. In addition to the high risk of the subway construction project itself, a large part of the the reason is the poor organization and management[4]. If the project management and coordination of various parties are not good, it wound not be able to ensure the construction quality and the control of unexpected risk factors[5].

However, the number of participants in the subway construction project is huge, and there are complex relationships among all stakeholders. Unclear responsibility allocation and the difficult of coordination between parties make the entire project management organization extremely complicated. However, the traditional project organization ignores the sociality, openness and network features of subway construction projects[6]. Therefore, research on project organizations needs to be placed in specific social networks. This paper uses social network, which provides a brand new perspective and method for studying the complex project organizational structure and relationship of subway construction projects. Through the network diagram, we can clearly and quantitatively analyze the network structure between the actors.

\section{Social Network Model Construction}

\subsection{Determination of Social Network Nodes}

Compared with the general construction projects, subway project construction is more complex. In addition to the construction process of the owner and contractor responsible for the construction process, it involves multiple levels of participants, including government departments, project intermediaries and so on. As the responsibility of the consulting unit in the construction of the subway construction project is to assist the risk management activities of the management department and 
provide advice, it doesn't participate in specific risk management activities, so it will not be included in the node range. Finally, 14 subway construction project participants are selected as network nodes, including owners, contractors, survey units, design units, supervision units, insurance companies, insurance brokers, public appraisers, third party monitoring units, suppliers, superior competent departments, relevant management departments, testing and evaluation units and environmental investigation units. Together, they form the core risk management participants in the entire process of the subway construction project in the construction phase. The effective management and control of risk management organizations will help the participants play their respective roles and the smooth implementation of subway construction projects.

\subsection{The Construction of Social Network Relationship Dimensions}

The strength of the relationship represents the degree of contact between the project participants. The closeness reflects the emotional dependence, resource sharing and communication between the participants. Scholars in different fields measure the relationship strength of social network from different dimensions. Poppo and Zenger define open communication, information sharing, trust, dependence and cooperation as indicators of informal relationship intensity[7].Xingzhi Liu measures the strength of actor's relationship from the four dimensions of trust, relationship commitment, cooperation and contact time[8].This article measures the strength of the relationship between project participants from the three dimensions of trust, dependence and communication, drawing on Xingzhi Liu's dimension of the relationship between the division of intensity and Combining with the characteristics of subway construction project organization.

Trust refers to believe each other to complete a particular behavior in the absence of supervision and control, and is willing to be in a state of possible harm. Therefore, only if the project participants trust each other can we ensure that the participants form a coordinated and cooperative partnership. The degree of dependence can reflect to some extent the intensity of the relationship between the parties. The dependence between participants can be manifested as dependency on tasks and dependence on resources. Communication can be measured both from the frequency and time of the communication between the two parties. The frequency of communication indicates the number of formal and informal contacts between both parties over the course of a given mission and the sharing of resources and information. The duration of communication also reflects the expected level of cooperation between the parties.

For the effective screening of the questionnaire, respondents from different units were classified according to the participants of the risk management organizations in the subway construction project. The relationship data of each participant in the questionnaire is collected. Finally, the collating data are input into the Ucinet 6.0 software to form the adjacency matrix of participants of the risk management organization.in the subway construction project. The network model of the risk management organization in subway construction project is shown in Figure 1.

\section{Network Structure Analysis of Risk Management Organization in Subway Construction Project}

There are many parameters to analyze the social network model. According to the analysis scope of the network structure index, this article analyzes the characteristics of the network model from the overall characteristics and individual characteristics to comprehensively and completely understand the organization's network structure. Among them, the overall analysis is the analysis of the overall network model, the individual network is to analyze the nodes in the network.

\subsection{Overall Network Characteristics Analysis}

\subsubsection{Network Density Analysis}

Network density analysis is done using Ucinet 6.0 software. According to the operation path of Network $\rightarrow$ Cohesion $\rightarrow$ Density, We import the converted binary data into Input Data and calculate the overall network density as shown in Figure 2. 


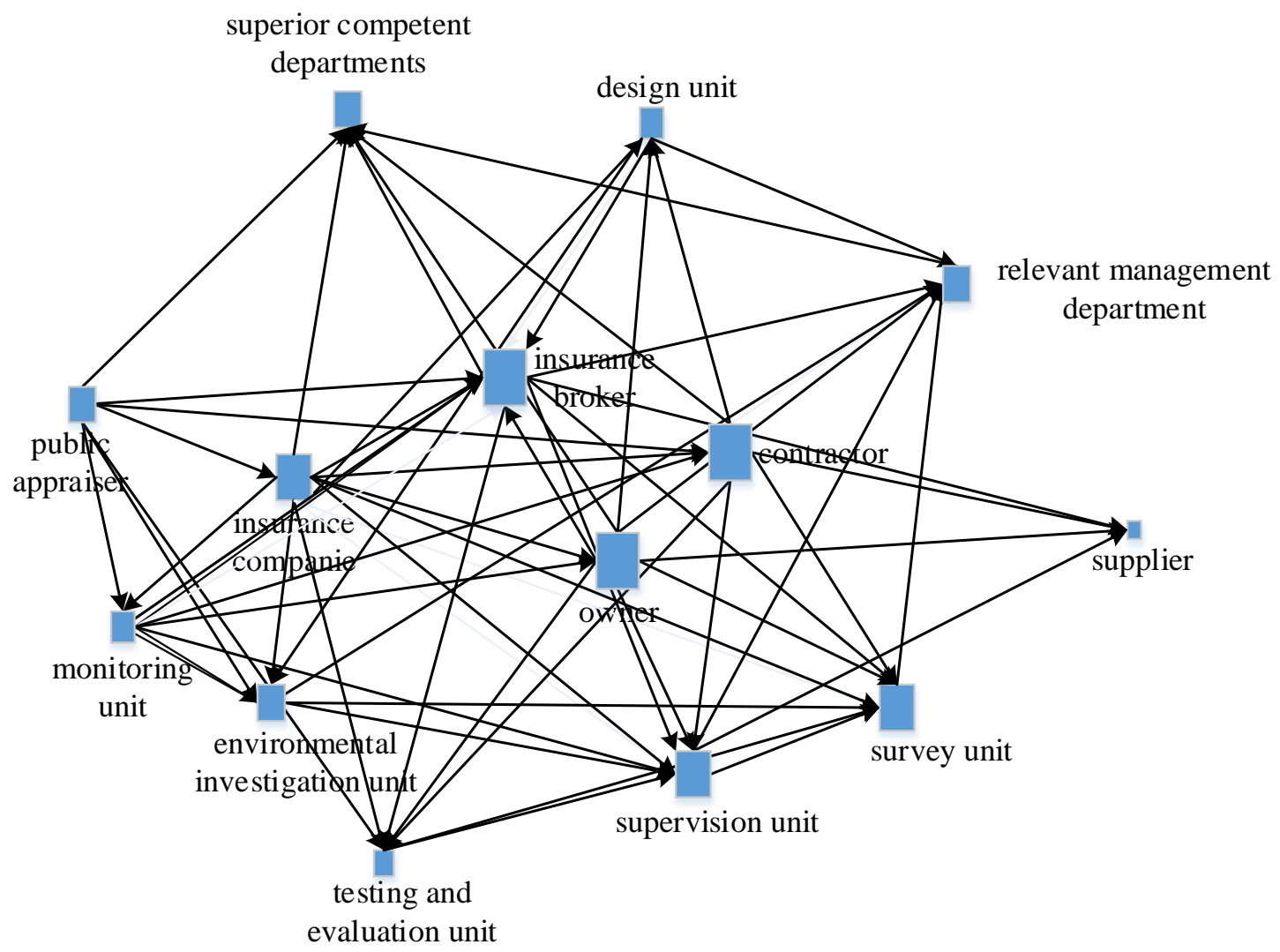

Figure 1. Social network model of risk management organization in subway construction project

\begin{tabular}{rr} 
Density & No. of Ties \\
\hline 0.2821 & 22.0000
\end{tabular}

Figure 2. Network density of risk management organization participant in subway construction project

The results show that the overall density is $0.2821 .28 .21 \%$ of the project risk management participants are closely related. Social network density is less than 0.5 and the value is low. On the one hand, it shows that there is a lack of communication among all the participants in the network. The frequency of interaction between participants is very low, which is not conducive to the sharing of network information and is most likely to cause the loss of risk control information. On the other hand, it shows that the rights and resources in the network are scattered. The lack of binding force on the parties is harmful to playing their role in the network for common risk management objectives.

\subsubsection{Network Centralization Analysis}

The center of the network is used to describe the overall centrality of the network and the degree which the entire network is clustered at a certain node. The center potential index calculated according to the Network $\rightarrow$ Centrality $\rightarrow$ Degree operation path, which is shown in Figure 3.

\section{Network Centralization $=60.26 \%$ \\ Heterogeneity $=10.17 \%$. Normalized $=3.26 \%$}

Figure 3. Network center potential of risk management organization in subway construction project

The network centralization is $60.26 \%$. It's more than $50 \%$. This shows that the central tendency of the entire network is more obvious with more concentrated distribution of rights and connections. 
The dominant players in the network may take advantage of differences in rights and decisions to increase their own advantage.

\subsection{Individual Network Characteristics Analysis \\ 3.2.1 Degree Centrality Analysis}

Owners, contractor and insurance brokers have the highest degree centrality index. They are all located at the core of risk management organization network in the subway construction project. Among them, the owners as the maker of the overall risk management objectives, all the construction units obey the overall goal and unified dispatch and command of the owners. The contractor is the main right party in the process of construction and is fully responsible for the safety risks in the construction process. The owners, contractors and insurance brokers all maintain strong and direct contacts with other participants in the network. They have a high degree of influence in the entire risk management organization and have advantages in risk control.

Third-party monitoring units, suppliers, assessors and testing and evaluation units have the lowest degree centrality. They are all on the edge of the network, which indicating that the information they owe is scarce and they are passive in receiving information from other parties. As a result, it is easy to cause information asymmetry and it is marginal in risk management organizations. There are few risk management activities in the participating networks, which is not conducive to the risk of the entire network Information Sharing. The four parties should strengthen their communication with other participants.

\subsubsection{Betweenness Centrality Analysis}

Among the networks with the highest center of gravity are owners and contractors. They are far beyond the other participants in the organization. This shows that the owner and the contractor occupy the important resources in the network and control the transmission of information in the network. At the same time, insurance brokers and supervision units's betweenness centrality are relatively high. Insurance brokers act as important resource sharing bridges and they have more resource control capability than other participants. The contractor, as the main responsibility unit for the risk management of the subway construction project, grasps the risk management and control information of each construction unit.

Each node in the relational network has great control over resources and the overall network is not stable enough. Insurance brokers and contractors with the highest Betweenness centrality are most likely to be structural holes in the network. They need to pay attention to their behavior in the network to prevent them from getting too much resources and failing to fully utilize the hysteresis and hindrances that cause resource transmission.

\subsubsection{Closeness Centrality Analysis}

The owners, contractors and insurance brokers have lower internal compactness and higher external compactness, and are close to all parties involved in the relationship. This shows that these parties have a great influence on other participants in the network and have strong control over other participants. At the same time, it is less dependent on other participants in the output of resources and is the key node in the network.

Many participants in the network, including testing and evaluation units, assessors, suppliers and third-party monitoring units, have higher internal compactness and the lower external compactness. Suggesting that these participants rely on other stakeholders for providing information and resources and they are in a passive position on the network and have little relationship with other participants in the network.

\section{Research Conclusion and Recommendations}

The entire network structure is not close enough, the network rights are mainly concentrated in the contractor. The network density of the entire project organization is low, the center is high, and the communication among the participants is relatively loose, and the rights in the network are concentrated in a few participants, including the owners, contractors and insurance brokers. These three parties have the highest centrality degree of network, occupy the core of the network and have strong resource control ability. 
Intermediaries have an important role to play, but their status in the network is low. Based on three central measurement indicators, except insurance brokers in the network, intermediaries including the public value, the third-party monitoring unit and the testing and evaluation unit, all have a low numerical centrality. The parties involved are not close enough to be at the edge of the network. The access to network resources is subject to the participation of owners, contractors and other parties, and their ability to control project risks is limited.

In order to enhance the cohesion of the project network and the connection between the parties involved, this paper puts forward the following suggestions: (1) Strengthen the contractor's comprehensive control of the risk in subway construction projects. (2)Encourage insurance companies to intervene in the risk management of subway construction projects in advance (3) Speed up the development and nurturing of risk management intermediaries in subway construction projects.

\section{References}

[1]. Zhiyuan Liu, Hui Che. Study on Risk Management Mode of Metro Engineering Construction Project [J]. Shanghai Insurance, 2017,(02):62-64.

[2]. Ninghui Liang, Xingrong Liu, Xueshan Cao et al. The Status and Development Strategy of Chinese Urban Subway Construction [J]. Journal of Chongqing Jianzhu University, 2008,(06):81-85.

[3]. Ru Mao. Actively carry out tunnel risk assessment and strengthen risk management [J]. Tunnel construction, 2007,(04):6+11.

[4]. Ronggui Ding, Fang Liu, Tao Sun et al. Project Management Based on Social Network Analysis -A Case Study of Large Construction Supervision Project [J]. China Soft Science, 2010, 06:132140.

[5]. Linjun Fan. Research on Network Modeling and Simulation of Large Engineering Project Network [D]. Changsha: National University of Defense Technology, 2010.

[6]. Yun Le, Dan Chong, Dongping Cao. Research on Construction Project Organization Based on Social Network Analysis [J]. Building economy, 2010,(08):31-33.

[7]. Poppo L., Zenger T. Do Formal Contracts and Relational Governance Function as Substitutes or Complements[J]. Strategic Management Journal, 2002, 23(8):707-725.

[8]. Xingzhi Liu. Research on Social Network Risk Analysis Methods of Project Management [D]. Shandong: Shandong University, 2011. 